\title{
Management of Cardiac Implantable Electronic Device Infection
}

\author{
Cristian Podoleanu¹ and Jean-Claude Deharo²
}

1. Cardiology Department, University of Medicine and Pharmacy Tirgu Mures, Tirgu Mures, Romania; 2. Cardiology Department, CHU La Timone, Marseille, France

\begin{abstract}
Despite improved preventive measures, infection associated with the use of cardiac implantable electronic devices (CIEDS) to treat often life-threatening conditions is rising at an average annual rate of almost $5 \%$. This rise is being driven by the increasing complexity of CIED technology and by the advancing age and co-morbidities of the patients. Although CIED infection is usually suspected based on local signs at the generator pocket site, diagnosis can be challenging in patients presenting no local manifestations or symptoms. Diagnostic methods include microbiological testing and echocardiography, and may be completed by positron emission tomography (PET)/computed tomography (CT) scan in selected cases. CIED infection requires a multidisciplinary approach in view of hardware extraction, targeted antibiotic therapy and reimplantation on an as-needed basis. Antibiotic prophylaxis targeting staphylococcal flora is recommended but the relation of these infections to medical care exposes patients to multi-resistant bacteria. New preventive measures utilising an antibacterial sleeve look promising. Treatment can be started on an empirical basis using an antistaphylococcal agent but must be continued using targeted antibiotic therapy. Crucial questions remain as to the best prevention strategy, optimal duration and timing of antibiotic therapy, and the most effective reimplantation technique.
\end{abstract}

\section{Keywords}

Infection, pacemaker, defibrillator

Disclosure: C Podoleanu has nothing to declare; JC Deharo has received honoraria for lectures from Spectranetics

Received: 11 July 2014 Accepted: 22 September 2014 Citation: Arrhythmia \& Electrophysiology Review, 2014;3(3):184-9 Access at: www.AERjournal.com

Correspondence: Jean-Claude Deharo, Cardiology Department, CHU La Timone, 264 Rue Saint Pierre, 13385 Marseille Cx 5, France. E: jean-claude.deharo@ap-hm.fr

The increasing use of cardiac implantable electronic devices (CIEDS) for management of cardiac conditions has over the last few years been associated with higher infection rates. ${ }^{1}$ Expanded CIED use alone cannot account for this rise, ${ }^{2-4}$ which involves both patient- and device-related factors. Indeed patients are tending to be older and presenting with co-morbidities, while devices are becoming more sophisticated and requiring more leads and revision. ${ }^{5}$

\section{Epidemiology}

Analysis of hospital discharge records including 4.2 million CIED implantations performed over the 16-year period from 1993 to 2008 showed that 69,000 patients required treatment for CIED infection. The average annual increase in CIED implantation was $4.7 \%$. Implantation of cardiac defibrillators accounted for half of the $96 \%$ overall increase in CIED implantation. The incidence of infection increased by $210 \%$ from 2,660 cases in 1993 to 8,230 cases in 2008. The annual rate of infection rose at a steady pace until 2004 when it jumped from $1.53 \%$ during that year to $2.41 \%$ in 2008 ( $p<0.001) .{ }^{6}$ In terms of patient demographics, the occurrence of CIED infection was greatest in white males over 65 years of age, and the most significant associated co-morbidities were renal failure, respiratory failure, heart failure and diabetes. The greatest risk factors for mortality were respiratory failure (odds ratio [OR] 13.58; $95 \%$ confidence interval [Cl] 12.88-14.3), renal failure (OR 4.28; $95 \% \mathrm{Cl} 4.04-4.53)$ and heart failure (OR 2.71; 95 \% Cl 2.54-2.88). ${ }^{6}$

These findings are in line with data from a national US survey ${ }^{2}$ including over 22,000 patients treated between 1996 and 2006. The study showed sharp increases in the proportion of CIED recipients with organ system failure (from $5.0 \%$ to $8.0 \%$ [p<0.001]) and with a diagnosis of diabetes mellitus (from $14.5 \%$ to $16.5 \%$ [p=0.005]). The mean age of patients presenting with CIED infection was $67 \pm 16$ years with a predominance of white patients (56\%) and males (66\%). The rise in CIED infection-related hospital admissions was not proportional to the increase in number of procedures.

A Danish population-based cohort study including more than 46,000 consecutive patients showed that the incidence rate of surgical site infection after pacemaker (PM) implantation was 4.82 per 1,000 PM-years (192 cases) after initial implantation and 12.12 per 1,000 PM-years (133 cases) after replacement. Independent factors associated with an increased infection risk were: number of operations including replacements, male sex, younger age, implantation during early study period and absence of antibiotics $(p<0.001)^{?}$

Analysis of the prospective REPLACE Registry (Complication Rates Associated With Pacemaker or Implantable Cardioverter-Defibrillator Generator Replacements and Upgrade Procedures) evaluating complications in patients who underwent CIED replacement at 72 US sites over six months revealed several interesting findings. ${ }^{8}$ The low infection rate of $1.3 \%$, was consistent with current practice including widespread use of antibiotic prophylaxis and other preventive measures. Post-operative haematoma was more frequent in patients who developed infection. Sites reporting higher infection rates had sicker patients and lower overall procedure volumes. 
Recently a risk evaluation score based on seven factors - early pocket reopening, male sex, diabetes, upgrade procedure, heart failure, hypertension and glomerular filtration rate $<60 \mathrm{~mL} / \mathrm{min}$ - was proposed. ${ }^{9}$ A retrospective analysis of 1,651 patients showed that scores ranged from 0 to 25 and identified three risk groups:

- Low: score 0-7 with $1.0 \%$ infection,

- Medium: score 8-14 with $3.4 \%$ infection,

- High: score $\geq 15$ with $11.1 \%$ infection.

It has recently been shown that ventricular assist device placement is also frequently complicated by infections. ${ }^{10}$ However, this specific problem is beyond the scope of this review.

\section{Diagnosis}

The clinical manifestations of CIED infection are highly variable. They can be divided into two somewhat overlapping categories, i.e. pocket infection and systemic infection. .112 $^{12}$ Pocket infection is usually due to surgical site contamination. Most cases occur within weeks or months following implantation but intervals of more than one year have been noted with micro-organisms such as Corynebacterium, Propionibacterium and certain species of coagulase-negative Staphylococci. Except in cases involving Staphylococcus aureus septicaemia, haematogenous contamination of the pocket from a distant focus is rare. ${ }^{13}$ Diagnostic suspicion of pocket infection is usually based on clinical symptoms such as local pain and discomfort due to inflammation of the generator pocket. In rare cases, patients with CIED infection may present with fever of unknown origin. Local signs may range from local redness to frank cutaneous erosion with exposure of the generator and/or leads (see Figure 1). ${ }^{4}$ In a study including 105 consecutive cases of infection, frank exposure occurred in 31 patients and infection with fistula, abscess or purulent collection occurred in 50 patients. The remaining patients presented inflammation or impending exteriorisation. ${ }^{14}$ A recent survey of European centres showed that the most common diagnostic features of pocket infection were pain, swelling and erythema the device pocket site. At most centres (68.1\%), these findings led to immediate hospitalisation for laboratory tests, echocardiographic examination and adequate treatment.' Appearance of skin pre-erosion over the can or lead is a common sign for suspicion of infection. One long-term 33-case study of outcomes after reimplantation of pulse generators showed that documented infection occurred in more than $50 \%$ of patients with local inflammatory manifestations or granulomatous-like changes involving the skin overlying the implantation site. The recommended treatment for these patients is to remove the pacing system before infection spreads to leads and cardiac tissue. The only cases in which infection did not occur after pocket revision involved patients in whom pre-erosion was due to mechanical causes. ${ }^{15}$

Systemic infections are usually due to haematogenous contamination in patients with multiple co-morbidities. The main sources of systemic infection are skin or soft tissue infections, pneumonia and bacteraemia associated with either intra-vascular catheters or invasive procedures. In most patients with systemic infection, blood cultures are positive, but prior antibiotic therapy can render cultures negative. Infective endocarditis is a common complication in patients with systemic infections, and lead or valve vegetations develop in nearly $25 \%$ of cases. ${ }^{11,12,16-18}$ Device-related endocarditis is often more insidious. Fever and chills are frequent when infection involves the intracardiac part of the PM or implantable cardioverter defibrillator

\section{Figure 1: Various Presentations of Pocket Infection.}
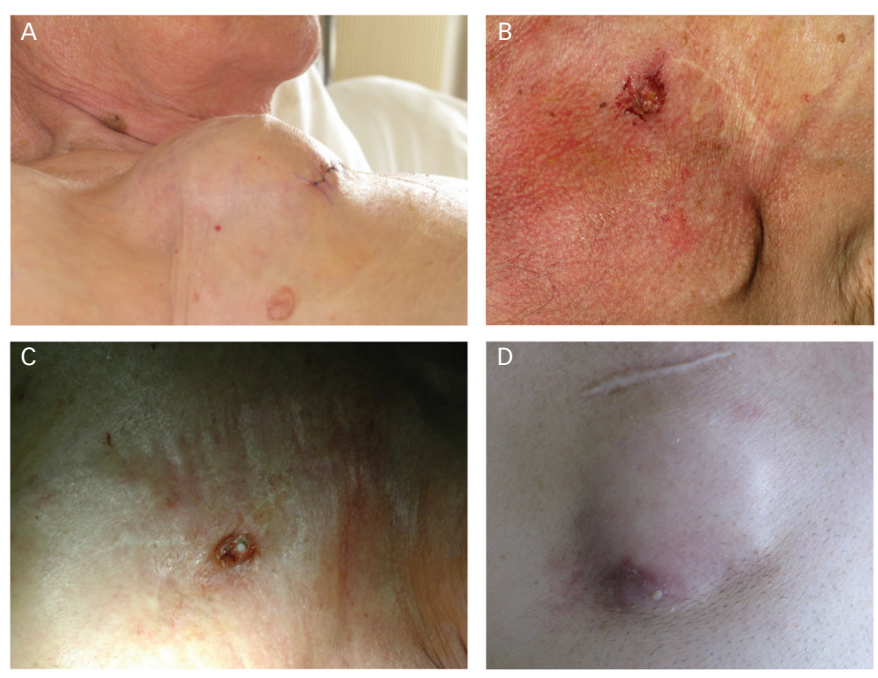

(A) Voluminous Collection Inside the Generator Pocket in a Patient Presenting Fever and Chills. (B) Pus and Erythematous Skin Overlying the Subcutaneous Route of a Lead. (C) Dehiscence of Infected Skin with Exposure of Lead Sleeve. (D) Redness and Thinning of Skin at Bottom of Pacemaker Pocket

(ICD) leads. In this situation, ultrasound examination may depict vegetations in the tricuspid valve..$^{19} \mathrm{~A}$ few patients may present with positive blood cultures with no evidence of pocket or lead infection. In these cases, the likelihood of CIED infection is greater if persistent unexplained bacteraemia is associated with Staphylococcus aureus than with gram-negative cocci. Another situation is the one of patients with CIED and infective endocarditis. Interestingly, a study in a population of PM recipients presenting infective endocarditis demonstrated three almost equally frequent infection scenarios characterised by: infection exclusively on PM leads, combination PM-lead and valvular infection (right or left valves), and isolated valvular infection apparently independent of PM leads. ${ }^{20}$

Several virulence factors contribute to the ability of micro-organisms to cause CIED infections. They include adherence factors, biofilm formation and microbial persistence. ${ }^{21}$ Staphylococci are the micro-organisms most frequently responsible for CIED infections. Other pathogens include other gram-positive cocci, Propionibacterium acnes and gram-negative bacilli. Figure 2 shows the distribution of pathogens encountered in patients managed for CIED infections at a tertiary European centre. Although there are no recent data specifically on the antibiotic sensitivity of micro-organisms causing CEID infections, ${ }^{18}$ the relation of these infections to medical care exposes patients to multi-resistant bacteria. Findings showing that contamination in some locations is associated with methicillin-resistant staphylococci in nearly $50 \%$ of cases ${ }^{11,12}$ justify empirical use of vancomycin pending positive identification of the organism. Gram-negative bacteria or polymicrobial infection are rare but probabilistic antibiotherapy covering gram-negative bacteria may be justified in patients with severe systemic infection. Fungal infections occur mainly in immunosuppressed patients.

Since identification of the pathogen responsible for the CIED infection is crucial for defining targeted antibiotherapy, at least two blood culture sets should be collected before starting antibiotics, and tissue from the explanted generator pocket and lead-tip should be cultured. Results should be interpreted in the light of the overall context since mistaken interpretation of positive cultures can unnecessarily prolong 
Figure 2: Pie Graph Showing Distribution of Infective Agents Identified at our Tertiary Centre in 197 Patients with Infected Cardiac Implantable Electronic Device

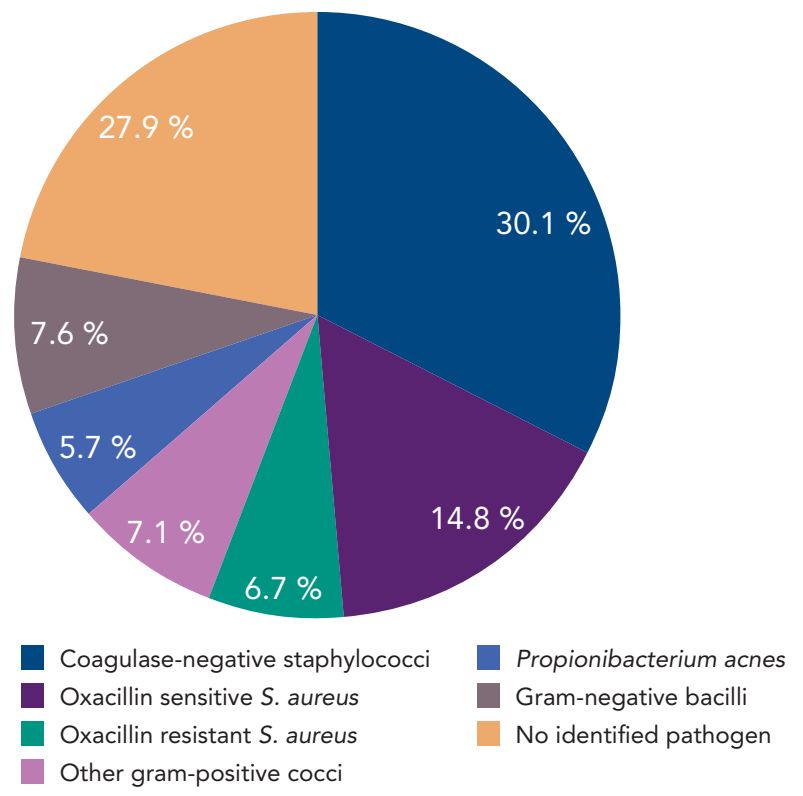

Note that no micro-organism was found in $27.9 \%$ of the patents, reflecting the high number of patients who had received antibiotics before referral. Source: Personal data published in Deharo et al., 2012.46

Figure 3: Ultrasound Image Showing 'Ghost' After Lead Extraction in a Patient with Cardiac Implantable Electronic Device Related Endocarditis - A Residual Tube-shaped Formation (arrow) Appears Along the Pathway of the Removed Lead

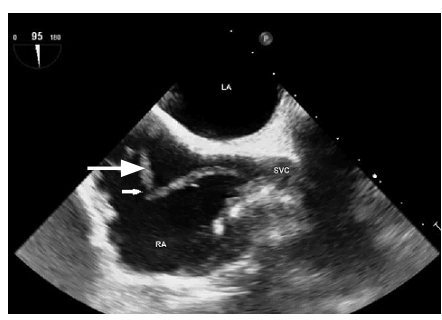

$L A=$ left atrium; $R A=$ right atrium; $S V C=$ superior vena cava

antibiotic therapy and increase related toxicities. ${ }^{3,22}$ In a large study of CIED infection, cultures of leads from 854 out of 1,204 (70.9\%) patients tested positive with isolation of a single micro-organism in 663 cases (77.6\%), two micro-organisms in 175 cases (20.5\%) and more than two micro-organisms in 14 cases (1.8\%). In 116 cases, material from the pocket was also cultured yielding results consistent with those from the leads in 69 cases (59 \%). Blood cultures performed in 359 cases were consistent with lead cultures in only 124 cases (35\%). Based on these findings, it was concluded that blood cultures are more likely to be contaminated and that monomicrobial CIED infection due to Staphylococcus species is common. ${ }^{23}$

The preferred technique for diagnostic imaging is transoesophageal echocardiography (TOE) because its sensitivity for detection of lead-related vegetations is greater than transthoracic echocardiography (TTE). Studies involving patients with endocarditis show that vegetations on the tricuspid valve or device lead were successfully detected in 90-96\% of cases with TOE as compared with only $22-43 \%$ of cases with TTE. ${ }^{20,23}$ Conversely, TOE is less effective than TTE for assessment of prognostic factors such as pericardial effusion, ventricular dysfunction, and dyssynchrony and pulmonary vascular pressure. ${ }^{4}$ Interestingly, a recent study carried out in a 212-patient cohort to determine the incidence, diagnostic value and outcome of intracardiac masses detected by echocardiography after device removal indicated that floating masses called 'ghosts' were observed in $8 \%$ of patients who underwent percutaneous device extraction following infection as compared with $0 \%$ of uninfected patients (see Figure 3). Based on these findings, it was concluded that presence of a ghost was indicative of device infection. ${ }^{24}$

In a multicentre feasibility study, intracardiac echocardiography (ICE) was compared with TOE for diagnosis of CIED-related endocarditis in 152 patients with endocarditis and 10 controls. Results showed that ICE was associated with better diagnostic yield..$^{25}$ The main limitations of TOE are poor detection of small intracardiac vegetations, occurrence of reverberation artifacts, and technical issues involving the distance between the probe and tricuspid valve. Another study indicated that ICE could be helpful in predicting the risks and complications of lead extraction procedures. ${ }^{26}$ Further study will be needed to confirm these preliminary results and to determine the situations in which the benefits of ICE might offset its invasiveness and higher cost.

A promising technique to enhance imaging of infected leads is ${ }^{18}$ F-fluorodeoxyglucose positron emission tomography/computerised tomography (FDG-PET/CT). In a pilot study of patients with suspected pacing system infections, PET scanning revealed lead infection in six out of 10 patients. ${ }^{27}$ Another study aimed at assessing the diagnostic yield of FDG-PET/CT in patients with cardiac CIED infection showed that the sensitivity and specificity of PET were $86.7 \%(59.5-98.3,95 \% \mathrm{Cl})$ and $100.0 \%(42.1-100,95 \% \mathrm{Cl})$, respectively, in patients with pocket site infection and $30.8 \%(9.1-61.4,95 \% \mathrm{Cl})$ and $62.5 \%(24.5-91.5$, $95 \% \mathrm{Cl}$ ), respectively, in patients with cardiac device-related infective endocarditis. ${ }^{28}$ FDG-PET/CT has also been useful for distinguishing CIED infection from recent post-implant changes and for guiding therapy. ${ }^{29}$

\section{Treatment and Prevention}

The general principles of CIED infection treatment involve effective antibiotic treatment, complete removal of the generator and leads, and implantation of a new system on an as-needed basis. ${ }^{4,30} \mathrm{~A}$ multidisciplinary approach with a team including, but not limited to, electrophysiologists, infectious disease specialists and cardiac surgeons is the key to successful management. Figure 4 shows the diagnostic and therapeutic approach currently used at a tertiary European centre.

The antibiotic of choice for initial empirical therapy is vancomycin because of its broad antistaphylococcal spectrum and of growing oxacillin-resistance. Alternatives include cefazolin or nafcillin in patients with oxacillin-susceptible staphylococcal strains. ${ }^{4}$ One report described the use of high-dose daptomycin therapy in 25 mostly elderly, male patients with large lead vegetations and severe co-morbidities. Administration of daptomycin at a median daily dosage of $8.3 \mathrm{mg} / \mathrm{kg}$ for a median duration of 20 days achieved an $80 \%$ clinical success rate and complete microbiological success in $92 \%$ of patients. ${ }^{31}$ After identification of the micro-organism, empirical antibiotherapy should be replaced by targeted treatment using the appropriate antibiotic defined on the basis of at least two independent blood cultures. In patients presenting infection confined to the pocket and subcutaneous tissue as documented by sterile blood cultures and negative result at TOE, ${ }^{4,11}$ targeted treatment must be continued for 10-14 days after hardware removal. In patients 


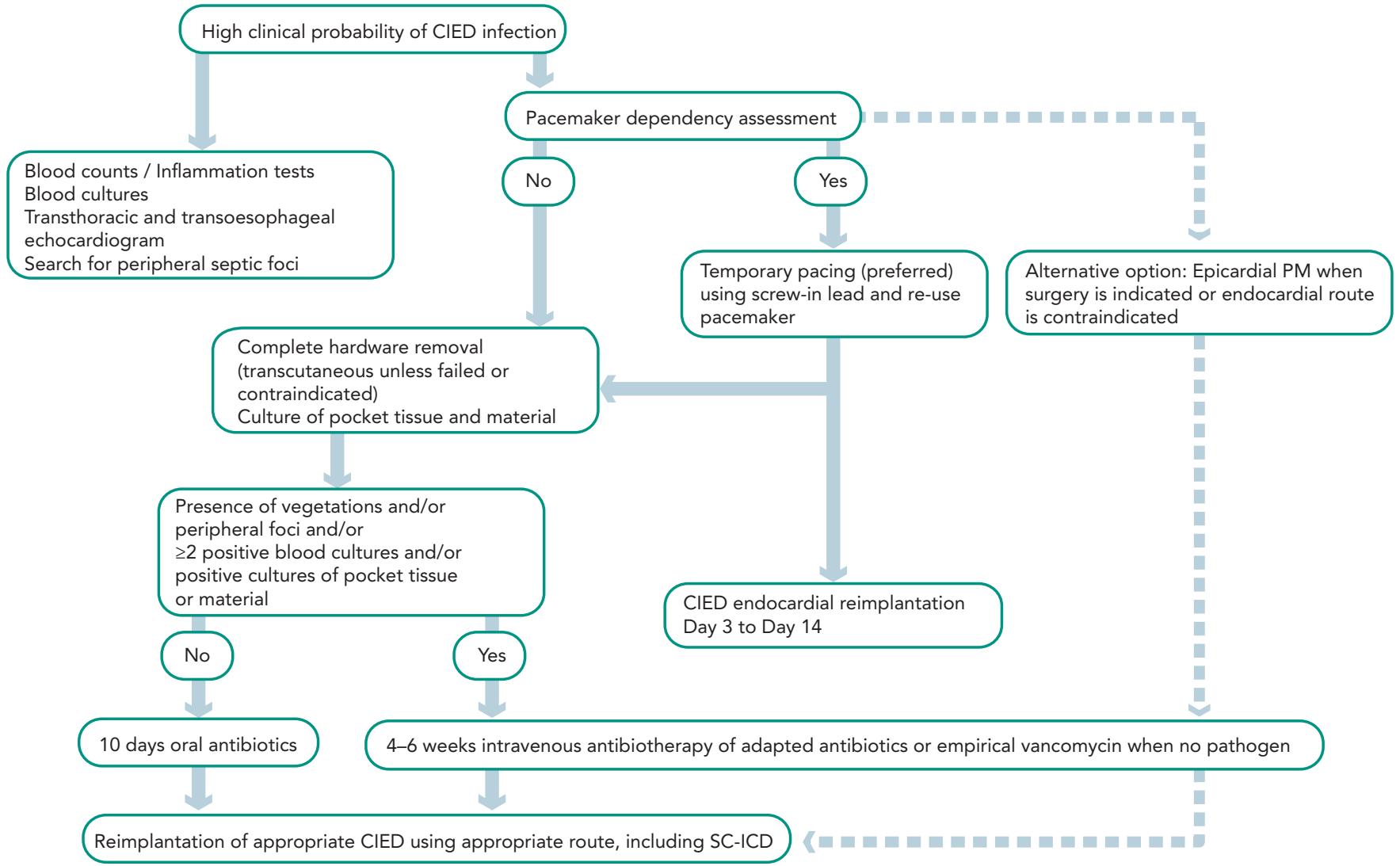

CIED = cardiac implantable electronic device; $P M=$ pacemaker; $S C-I C D=$ subcutaneous implantable cardioverter defibrillator

presenting documented CEID-related endocarditis or positive bacteraemia, targeted intravenous antibiotherapy must be continued for 4-6 weeks. ${ }^{4}$

Total CIED removal is mandatory for patients presenting documented valvular and/or lead endocarditis or sepsis, pocket infection with abscess formation, device erosion or valvular endocarditis without definite involvement of the CIED. ${ }^{32}$ Early hardware removal has been associated with improved outcomes but complications cannot be ruled out. In a retrospective study including 416 patients with CIED infection, 44 (12.0\%) developed complications related to device removal and three $(0.8 \%)$ died. Although device removal complications have been linked with higher mortality at 30 days and at one year, comparison of patients undergoing immediate removal with patients undergoing delayed or no removal has demonstrated a three-fold decrease in one-year mortality. ${ }^{33}$ In a cohort study including 177 patients with CIED endocarditis, the one-year mortality rate was $19.9 \%$ for patients who underwent device removal during hospitalisation versus $38.2 \%$ for patients who did not undergo device removal. ${ }^{34}$ Conservative management of the infected CIED has been reported in small case series. This approach should only be used if the risk of extraction outweighs the potential benefit, i.e. for patients whose condition contraindicates the removal procedure or whose life expectancy is short due to co-morbidity or advanced age..$^{35}$ In rare cases where infection is superficial or confined to the incisional scar, it may be possible to leave the device in situ and administer oral antibiotics for 7-14 days. ${ }^{4}$ At the clinical level, however, these patients are difficult to differentiate from patients presenting early deep infection and require close follow-up after treatment.
Prophylaxis with systemic antibiotics targeting staphylococcal flora is strongly recommended before implantation of a CIED because of the risk of contamination by micro-organisms on normal skin. 18,30,36 $\mathrm{A}$ metaanalysis comprising 2,023 patients showed that administration of antibiotic prophylaxis, mainly using antistaphylococcal penicillins and first-generation cephalosporins, immediately before permanent PM implantation consistently achieved a protective effect (OR 0.256; $95 \% \mathrm{Cl} 0.10-0.656$ ). ${ }^{30}$ Most experts advocate use of first-generation cephalosporins such as cefazolin for systemic prophylaxis. A study including 852 patients described use of an intravenous course of $2 \mathrm{~g}$ cefazolin 20 minutes before new permanent PM implantation or pulse generator replacement. Analysis of early phase outcomes showed minor complications in nine patients (1.0\%). During long-term follow-up, major infectious complications were observed in six patients $(0.7 \%){ }^{37} \mathrm{~A}$ randomised controlled prospective study comparing CIED implantation with or without cefazolin prophylaxis was undertaken but prematurely discontinued after reaching $65 \%$ of the planned enrolment, due to appearance of a significant difference in favour of the antibiotic arm ( 0.63 versus 3.28 infected patients, $p=0.016){ }^{38}$ Although vancomycin is not generally recommended, it may be considered as an alternative at centres where oxacillin resistance is high. ${ }^{4}$

With no evidence documenting its efficacy, administration of post-operative antibiotic therapy is not recommended due to its potential association with adverse side-effects and selection of drug resistant organisms. ${ }^{4,8}$

Recently a new technique to reduce surgical site infections was proposed. The CIED is implanted within an antibacterial polypropylene mesh sleeve that releases minocycline and rifampicin in the generator 
Figure 5: Photo and X-ray Illustrating Temporary Pacing in a Pacemaker-dependent Patient Using a Right Ventricular Screw-in Lead Introduced via the Left Internal Jugular Vein and Connected to a Re-use Pacemaker Taped on the Thorax
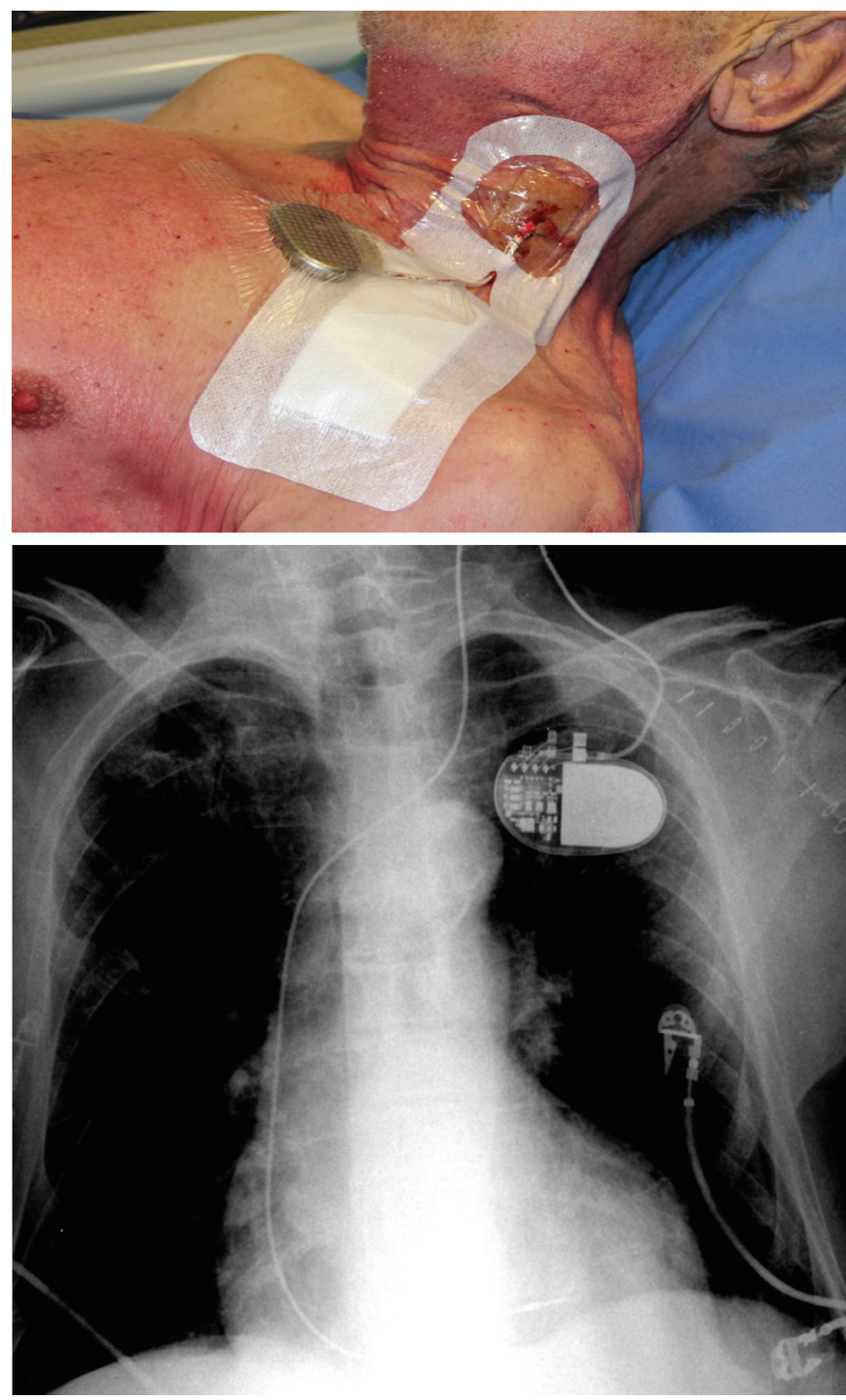

pocket. Preclinical and small-scale clinical studies suggest that this technique can reduce CIED infection in high-risk patients. ${ }^{9,39-41}$ Prospective registries are ongoing and randomised studies are warranted to validate routine use of this promising prophylactic tool.

\section{Reimplantation of a Device After Infection}

Reimplantation is a major concern in patients treated for CIED infection. Before undertaking reimplantation, a careful individual assessment should be carried out to confirm if there is a continued need for a new CIED. ${ }^{4,32,42}$ A retrospective review indicated that reimplantation was unnecessary in one-third of cases. ${ }^{11}$ Another report described a study carried out in 188 patients to test the safety of deferring PM after CIED removal for infection. Selection of patients eligible for deferring reimplantation was based on electrocardiographic and monitoring data. Assessment of outcomes after a 12-month follow-up period indicated that these data were useful in distinguishing patients for whom reimplantation could be safely deferred from patients requiring a new implant. ${ }^{43}$ In contrast with these findings, a study on 151 patients who underwent extraction of biventricular pacing devices for infection-related complications indicated that outcomes were worse in patients deemed cured after removal but not reimplanted than in patients with successful cardiac resynchronisation therapy (CRT) reimplantation. ${ }^{44}$

When needed, replacement device implantation should be carried out in an alternative location such as the contralateral side, iliac vein or epicardial implantation. With regard to timing, it seems reasonable to postpone reimplantation until infection has resolved, but there are no clear guidelines. ${ }^{4,12,45}$ Relevant decisionmaking factors include presence of bacteraemia or sterile blood cultures before and after removal, and type of the identified pathogen strain. ${ }^{4,1,1,12}$ In the literature, intervals for reimplantation have ranged from 24 hours to 14 days. ${ }^{4}$ For PM-dependent patients, temporary pacing is an option ${ }^{4}$ but it is not recommended ${ }^{45}$ and has the risk of malfunction and short-term capability. Tarakji et al. ${ }^{12}$ recently reported that the recurrent infection rate was higher in patients in whom device extraction and reimplantation were performed during the same hospital stay. This finding suggests that a waiting period is required for safe reimplantation. Epicardial pacing is an option but has been associated with higher mortality rates. ${ }^{46}$ An interesting alternative is temporary pacing using a screw-in pacing lead connected to a re-use can strapped on the skin of the patients, ${ }^{47,48}$ the so called 'semi-permanent' pacing (see Figure 5). This approach allows patients to safely await implantation of a new device for the recommended 72 hours to 14 days depending on clinical status.

\section{Outcomes}

CIED infection is a severe medical condition causing significant morbidity and mortality. Despite adequate treatment, reported death rates range from $8.0 \%$ to $26.9 \% \%^{4,5,17,49-52}$ Several predictors of long-term mortality have been identified including older age, heart failure, infective endocarditis, renal failure and long-term corticosteroid therapy. ${ }^{46,53}$ However, these high mortality rates must be seen in the context of populations with multiple co-morbidities. Indeed, device patients have shown high mortality rates. ${ }^{54-56}$

While the overall benefits of device removal are unquestionable, ${ }^{4}$ performing the procedure using a multidisciplinary approach employing device extraction and directed antibiotic therapy is probably crucial to decreasing mortality. ${ }^{46}$ In a prospective cohort study designed to evaluate a systematic approach to managing CIED infections, a total of 194 patients admitted for CIED infection between 2004 and 2008 were enrolled and matched by age, sex and type of device to a cohort of uninfected CIED patients. ${ }^{46}$ Results at one-year showed that all-cause long-term mortality in the study cohort was not significantly different from controls $(14.3 \%$ versus $11.0 \%$, respectively) despite an initial in-hospital mortality rate of $4.1 \%$ in the study cohort. Similarly the one-year mortality rate was not significantly different in patients with pocket infection versus infective endocarditis (12.5\% versus $15.5 \%$, respectively). The only factors associated with long-term mortality in patients with infected CIED were older age, CRT device infection, thrombocytopaenia (platelet count $<100$ giga/litre at admission) and renal dysfunction (serum creatinine $>150 \mathrm{mmol} /$ litre). Coagulase-negative staphylococci were associated with a lower risk of death. Fourteen (11.2 \%) of the 125 patients who underwent device reimplantation died. After adjusting for the previously identified predictors (i.e. age, CRT device infection, thrombocytopaenia and coagulase-negative staphylococci) reimplantation with an epicardial right ventricular pacing system was 
significantly associated with mortality (hazard ratio [HR] 2.85, $95 \%$ Cl 1.08-7.50, $p=0.034)$. Twelve of 14 (85.7\%) patients who died had been reimplanted with epicardial systems as compared with 54 of 111 (48.6\%) survivors.

In another study that enrolled 189 patients with CIED infection, treatment consisted of immediate removal in $183(96 \%)$, removal after failure of medical therapy in three $(2 \%)$ and parenteral antimicrobial therapy in all patients. ${ }^{11}$ In-hospital mortality after a mean follow-up of 175 days was $3.7 \%$. During follow-up after discharge, relapse or persistent pocket infection was noted in $5 \%$ of patients while the remaining $95 \%$ were infection-free at the end of the study period.

\section{Conclusion}

Infection of CIED is a growing problem. In any form, CIED infection is a severe complication requiring hardware extraction and targeted antibiotic therapy in most cases. Further study is necessary to determine the best prevention strategy, optimal duration and timing of antibiotic therapy, and most effective guidelines for reimplantation.
1. Bongiorni MG, Marinskis G, Lip GY, et al. How european centres diagnose, treat, and prevent CIED infections: Results of an European

2. Voigt $A$, Shalaby $A$, Saba $S$. Continued rise in rates of cardiovascular implantable electronic device infections in the united states: Temporal trends and causative insights. Pacing Clin Electrophysiol 2010;33:414-9.

3. Baddour LM. Cardiac device infection-or not. Circulation 2010;121:1686-7.

4. Baddour LM, Epstein AE, Erickson CC, et al. Update on cardiovascular implantable electronic device infections and their management: A scientific statement from the American Heart Association Circulation 2010:121:458-77.

5. Baman TS, Gupta SK, Valle JA, Yamada E. Risk factors for mortality in patients with cardiac device-related infection. Circ Arrhythm Electrophysiol 2009;2:129-34.

6. Greenspon AJ, Patel JD, Lau E, et al. 16-year trends in the infection burden for pacemakers and implantable cardioverterdefibrillators in the United States 1993 to 2008. I Am Coll Cardiol 2011;58:1001-6.

7. Johansen JB, Jørgensen OD, Møller M, et al. Infection after pacemaker implantation: infection rates and risk factors associated with infection in a population-based cohort study of 46299 consecutive patients. Eur Heart I 2011;32:991-8.

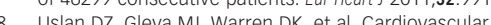
implantable electronic device replacement infections and prevention: results from the REPLACE Registry. Pacing Clin Electrophysiol 2012;35:81-7.

9. Mittal S, Shaw RE, Michel K, et al. Cardiac implantable electronic device infections: Incidence, risk factors, and the effect of the AigisRx antibacterial envelope. Heart Rhythm 2014;11:595-601.

10. Gordon RJ, Weinberg AD, Pagani FD, et al. Prospective, multicenter study of ventricular assist device infections. circulation 2013;127:691-702.

11. Sohail MR, Uslan DZ, Khan AH, et al. Management and outcome of permanent pacemaker and implantable cardioverter-defibrillator infections. J Am Coll Cardio 2007:49:1851-9.

12. Tarakji KG, Chan EJ, Cantillon DJ, et al. Cardiac implantable electronic device infections: Presentation, management, and patient outcomes. Heart Rhythm 2010;7:1043-7.

13. Chamis AL, Peterson GE, Cabell CH, et al. Staphylococcus aureus bacteremia in patients with permanent pacemakers or implantable cardioverter-defibrillators. Circulation 2001:104:1029-33.

14. Klug $D$, Wallet $F$, Lacroix $D$, et al. Local symptoms at the site of pacemaker implantation indicate latent systemic infection Heart 2004;90:882-6.

15. Cassagneau R, Ploux S, Ritter P, et al. Long-term outcomes after pocket or scar revision and reimplantation of pacemakers with preerosion. Pacing Clin Electrophysiol 2011;34:150-4.

16. Le KY, Sohail MR, Friedman PA, et al. Clinical predictors of cardiovascular implantable electronic device-related infective endocarditis. Pacing Clin Electrophysiol 2011:34:450-9.

17. Sohail MR, Uslan DZ, Khan AH, et al. Infective endocarditis complicating permanent pacemaker and implantable cardioverter-defibrillator infection. Mayo clin Proc 2008;83:46-53.

18. Klug $D$, Balde $M$, Pavin $D$, et al. Risk factors related to infections of implanted pacemakers and cardioverterdefibrillators: results of a large prospective study. Circulation 2007:116:1349-55

19. Le Dolley $\mathrm{Y}$, Thuny $F$, Bastard $E$, et al. Images in cardiovasclar medicine: pacemaker lead vegetation trapped in patent foramen ovale: a cause of hypoxemia after percutaneous foramen ovale: a cause of hypoxemia a
20. Duval X, Selton-Suty C, Alla F, et al. Endocarditis in patients with a permanent pacemaker: a 1-year epidemiologica survey on infective endocarditis due to valvular and/or pacemaker infection. Clin Infect Dis 2004:39:68-74.

21. Nagpal A, Baddour LM, Sohail MR. Microbiology and pathogenesis of cardiovascular implantable electronic device infections. Circ Arrhythm Electrophysiol 2012;5:433-41.

22. Pichlmaier M, Marwitz V, Kühn C, et al. High prevalence of asymptomatic bacterial colonization of rhythm managemen devices. Europace 2008;10:1067-72.

23. Bongiorni MG, Tascini C, Tagliaferri E, et al. Microbiology of cardiac implantable electronic device infections. Europace 2012;14:1334-9

24. Le Dolley Y, Thuny F, Mancini J, et al. Diagnosis of cardiac device-related infective endocarditis after device removal. JACC Cardiovasc Imaging 2010;3:673-81.

25. Narducci ML, Pelargonio G, Russo E, et al. Usefulnes of intracardiac echocardiography for the diagnosis of cardiovascular implantable electronic device-related endocarditis. J Am Coll Cardiol 2013;61:1398-405.

26. Bongiorni MG, Di Cori A, Soldati E, et al. Intracardiac echocardiography in patients with pacing and defibrillating leads: A feasibility study. Echocardiography 2008:25:632-8.

27. Ploux S, Riviere A, Amraoui S, et al. Positron emission tomography in patients with suspected pacing system infections may play a critical role in difficult cases. Heart Rhythm 2011:8:1478-81.

28. Cautela J, Alessandrini S, Cammilleri S, et al. Diagnostic yield of FDG positron-emission tomography/computed tomography in patients with CEID infection: a pilot study. Europace 2013;15:252-7

29. Sarrazin JF, Philippon F, Tessier M, et al. Usefulness of fluorine-18 positron emission tomography/computed tomography for identification of cardiovascular implantable electronic device infections. J Am Coll Cardiol 2012;59:1616-25.

30. Baddour LM, Cha YM, Wilson WR. Clinical practice. Infections of cardiovascular implantable electronic devices. N Eng/ J Med 2012:367:842-9.

31. Durante-Mangoni E, Casillo R, Bernardo M, et al. High-dose daptomycin for cardiac implantable electronic device-relate infective endocarditis. Clin Infect Dis 2012;54:347-54.

32. Wilkoff BL, Love CJ, Byrd CL, et al. Transvenous lead extraction: Heart Rhythm Society expert consensus on facilities, training, indications, and patient management: This document was endorsed by the American Heart Association (AHA). Heart Rhythm 2009:6:1085-104

33. Le KY, Sohail MR, Friedman PA, et al Impact of timing of device removal on mortality in patients with cardiovascular implantable electronic device infections. Heart Rhythm 2011;:8:1678-85

34. Athan $\mathrm{E}, \mathrm{Chu} \mathrm{VH}$, Tattevin $\mathrm{P}$, et al. Clinical characteristics and outcome of infective endocarditis involving implantable cardiac devices. JAMA 2012;307:1727-35.

35. Lopez JA. Conservative management of infected pacemaker and implantable defibrillator sites with a closed antimicrobia irrigation system. Europace 2013:15:541-5.

36. Da Costa $\mathrm{A}$, Lelièvre $\mathrm{H}$, Kirkorian $\mathrm{G}$, et al. Role of the preaxillary flora in pacemaker infections: a prospective study. Circulation 1998:97:1791-5.

37. Bertaglia E, Zerbo F, Zardo $S$, et al. Antibiotic prophylaxis with a single dose of cefazolin during pacemaker implantation: Incidence of long-term infective complications. Pacing Clin Electrophysiol 2006;29:29-33

38. de Oliveira JC, Martinelli M, Nishioka SA, et al. Efficacy of antibiotic prophylaxis before the implantation of pacemakers and cardioverter-defibrillators: results of a large, prospective, randomized, double-blinded, placebo-controlled trial. Circ Arrhythm Electrophysiol 2009:2:29-34.

39. Hansen $L K$, Brown M, Johnson D, et al. In vivo model of human pathogen infection and demonstration of efficacy by an antimicrobial pouch for pacing devices. Pacing Clin Electrophysiol 2009:32:898-907.

40. Bloom HL, Constantin L, Dan D, et al. Implantation success and infection in cardiovascular implantable electronic device procedures utilizing an antibacterial envelope. Pacing Clin Electrophysiol 2011;34:133-42.

41. Kolek MJ, Dresen WF, Wells QS, Ellis CR. Use of an antibacterial envelope is associated with reduced cardiac implantable electronic device infections in high-risk patients. Pacing Clin Electrophysiol 2013;36:354-61.

42. Deharo JC, Bongiorni MG, Rozkovec A, et al. Pathways for training and accreditation for transvenous lead extraction: a European Heart Rhythm Association position paper. Europace 2012:14:124-34.

43. Marijon $E$, De Guillebon $M$, Bordachar $P$, et al. Safety of deferring the reimplantation of pacing systems after their removal for infectious complications in selected patients: a 1-year follow-up study. J Cardiovasc Electrophysiol 2010;21:540-4

44. Rickard J, Tarakji K, Cheng A, et al. Survival of patients with biventricular devices after device infection, extraction, and reimplantation. JACC Heart Fail 2013;1:508-13.

45. Habib G, Hoen B, Tornos P, et al. Guidelines on the prevention, diagnosis, and treatment of infective endocarditis (new version 2009): the Task Force on the Prevention, Diagnosis, and Treatment of Infective Endocarditis of the European Society of Cardiology (ESC). Endorsed by the European Society of Cardiology (ESC). Endorsed by the European Society of Clinical Microbiology and Infectious Diseases (ESCMID) and the International Society of
Chemotherapy (ISC) for Infection and Cancer. Eur Heart Chemotherapy (ISC)
2009:30:2369-413.

46. Deharo JC, Quatre A, Mancini J, et al. Long-term outcomes following infection of cardiac implantable electronic devices: A prospective matched cohort study. Heart 2012;98:724-31.

47. Braun MU, Rauwolf $\mathrm{T}$, Bock M, et al. Percutaneous lead implantation connected to an external device in stimulationdependent patients with systemic infection--a prospective and controlled study. Pacing Clin Electrophysiol 2006;29:875-9.

48. Kawata H, Pretorius V, Phan $H$, et al. Utility and safety of temporary pacing using active fixation leads and externalized re-usable permanent pacemakers after lead extraction. Europace 2013;15:1287-91.

49. Uslan DZ, Sohail MR, St Sauver JL, et al. Permanent pacemaker and implantable cardioverter defibrillator infection: a population-based study. Arch intern Med 2007;167:669-75

50. Chua JD, Wilkoff BL, Lee I, et al. Diagnosis and management of infections involving implantable electrophysiologic cardiac devices. Ann Intern Med 2000;133:604-8.

51. Cacoub $P$, Leprince $P$, Nataf $P$, et al. Pacemaker infective endocarditis. Am I Cardiol 1998;82:480-4.

52. Klug D, Lacroix D, Savoye C, et al. Systemic infection related to endocarditis on pacemaker leads: Clinical presentation and management. Circulation 1997;95:2098-107.

53. Habib A, Le KY, Baddour LM, et al. Predictors of mortality in patients with cardiovascular implantable electronic device infections. Am I Cardiol 2013:111:874-9.

54. Brunner M, Olschewski M, Geibel A, et al. Long-term survival after pacemaker implantation. Prognostic importance of gender and baseline patient characteristics. Eur Heart 」 2004;25:88-95.

55. Cleland JG, Daubert JC, Erdmann E, et al. The effect of cardiac resynchronization on morbidity and mortality in heart failure. N Engl J Med 2005;352:1539-49.

56. Goldenberg I, Gillespie J, Moss AJ, et al. Long-term benefit of primary prevention with an implantable cardioverterdefibrillator: an extended 8-year follow-up study of the Multicenter Automatic Defibrillator Implantation Trial II. Circulation 2010;122:1265-71. 